\title{
VESTIR AL OTRO EN EL CARIBE. ROPAS PARA INDIOS Y POBRES
}

\section{Dress the Other in the Caribbean. Clothing for Indians and the Poor}

\section{Roberto Valcárcel Rojas ${ }^{1}$}

\section{Resumen}

La desnudes indígena fue enfrentada desde el proyecto de evangelización y civilización desplegado por España en el Nuevo Mundo. No solo se trató de una supuesta confrontación ética y moral sino de un acto de dominación en el que imponer la norma de vestir a los indígenas, servía para atacar sus identidades originarias y construir un nuevo ser, el indio. El uso y comercio de la ropa de segunda mano funciona varios siglos después como un recurso de señalización y explotación de la pobreza. En este texto reflexiono sobre estos dos momentos donde la ropa sirve para construir la identidad y naturaleza de los subordinados, y apuntar sobre la ubicación marginal del Caribe en los diseños de estructuración del poder global. A través del análisis histórico sobre manejo de ropa entre indígenas de las Antillas Mayores, y la vivencia antropológica en espacios comerciales de la ciudad de Santo Domingo, República Dominicana, se constata la reiteración de los mecanismos de dominio y el papel central de la cultura material en estos.

Palabras claves: Indio; indígenas; Caribe; ropa de segunda mano

1. Investigador Posdoctoral en la Universidad de Leiden.Profesor invitado del Instituto Tecnológico de Santo Domingo (INTEC), República Dominicana. Email: rv.rojas68@yahoo.es "[los indios]... son buenos para les mandar, y les hacer trabajar, sembrar y hacer todo lo otro que fuere menester, y que hagan villas, y se enseñen a andar vestidos y a nuestras costumbres. Estas son sus palabras formales del Almirante".

Fray Bartolomé de Las Casas (1875, p. 377)
The indigenous nudity was faced from the project of evangelization and civilization develop by Spain in the New World. It was not only an alleged ethical and moral confrontation but an act of domination in which dressing the indigenous, served to attack their original identities and build a new being, the Indian. The use and trade of second-hand clothing functions several centuries later as a resource for signaling and exploitation of poverty. In this text I reflect on these two moments where clothing serves to build the identity and nature of subordinates, and to show the marginal location of the Caribbean in the designs of global power. Through the historical analysis on the use of clothing among indigenous people of the Greater Antilles, and the anthropological experience in commercial spaces of Santo Domingo city, Dominican Republic, it is verified the reiteration of the mechanisms of domination and the central role of material culture in these.

Keywords: Indian; Indigenous; Caribbean; second-hand clothing. 
Entre sus múltiples sorpresas, el Nuevo Mundo ofreció a los europeos un escenario de gentes desnudas. Como pecaminoso, lascivo e ignorante de una mínima civilidad, se consideró este hábito indígena, en el que otros vieron la naturaleza sencilla de gente inocente y carente de maldad. Cubrirse el cuerpo solo con ornamentos, pinturas corporales y, en ocasiones, mínimas prendas y objetos destinados a proteger los genitales, definía un modo particular de interactuar con el clima y la naturaleza caribeña, de expresar percepciones estéticas y rituales, visiones del cuerpo y su simbolismo construidas a lo largo de siglos de vida en ambientes tropicales.

El proyecto colonial atacó la desnudez en cualquiera de sus expresiones. El vestuario definido desde una perspectiva occidental, se impuso como herramienta de subordinación y construcción del individuo dominado. Como ha dicho Enrique Dussel (1994), el descubrimiento fue encubrimiento pues el Otro, el vencido, fue ocultado y transformado. Literalmente, la ropa que cubrió estos cuerpos sometidos y oprimidos ayudó en tan lamentable tarea.

Quinientos ańos después, las ropas han adquirido un protagonismo que no habían tenido en la región desde aquellos días de fundación colonial. Las prendas desechadas por los poderosos o sus sistemas de consumo, hacen un recorrido planetario para anclarse en el lado pobre del mundo. Vuelven como parte de un negocio global que, entre sus muchas facetas, señaliza la miseria tanto por el uso de esta ropa como por su comercialización. Son indumentarias signadas, como las entregadas generalmente a los indios, por el hecho de estar destinadas a gente secundaria, prescindible. En este texto reflexionamos sobre estos dos momentos donde la ropa sirve para construir la identidad y naturaleza de los subordinados, y apuntar sobre la ubicación marginal del Caribe en los diseńos de estructuración del poder global. Nos movemos entre el análisis histórico y la vivencia antropológica, para constatar la reiteración de los mecanismos de dominio y el papel central de la cultura material en estos.

\section{La paga de los caciques Yaguax y Caicedo}

Ciudades, iglesias, fuertes, estancias, casas de paja y piedra, fueron levantadas por los indígenas en la isla de La Española, corazón del esfuerzo colonial en el Caribe. En 1506, en pago por su labor en las obras de la ciudad de Santo Domingo, se dio a los caciques Yaguax y Caicedo, y su gente, " 24 camisas de lienzo labradas y unas naguas de paño y tres bonetes colorados de grana y dos sombreros guarnecidos y dos pares de caraguelles (sic)" (Mira Caballo 2000, p. 107). La ropa, inicialmente, llegó a los indígenas -junto a cascabeles y cuentas de vidrio- como regalo o elemento de trueque, parte de un mundo exótico y nuevo cuya materialidad querían captar. Para 1506 se había convertido en medio de retribución del trabajo que se veían obligados a realizar pero, sobre todo, en elemento del accionar de civilización y aculturación destinada a transformar al indígena en "indio": un ser obligado a seguir las normas de apariencia, vida y credo del mundo colonial, donde se abandonan las viejas prácticas identitarias -como las modificaciones y pinturas corporales-, se adquiere una nueva lengua y se diluyen las antiguas pertenencias étnicas.

Como parte de la encomienda, sistema que obligaba a una comunidad o grupo de indígenas, a trabajar para un español durante varios meses al año a cambio de supuesta instrucción civilizatoria y religiosa, se ordenó al encomendero proporcionar vestimenta o los medios para comprarla. El pago de un peso de oro al año, a usar en la adquisición de ropas, fue establecido en las Leyes de Burgos de 1512. Para los caciques y sus mujeres se preveía una cantidad de dinero mayor a fin de darles un ajuar más completo y adecuado a su rango. En la moderación a las leyes (1513) se mandaba que, en dos años, ya todos los hombres y mujeres debían andar vestidos (Muro 1956, p. 62). 
El padre Bartolomé de Las Casas comenta la imposibilidad de vestir a los indios con tan reducidos fondos. De cualquier modo se fomentó la práctica, aun cuando en el interrogatorio jeronimiano de 1517 se critica la baja calidad de los vestidos. Eran fabricados de lienzo delgado y usados por los indios hasta podrírseles sobre el cuerpo (Mira Caballos 1997, p. 137). Según Gonzalo Fernández de Oviedo, con el tiempo el uso de ropa comenzó a generalizarse y, hacia la década del treinta del siglo XVI, la mayoría de los indios en estancias y pueblos de La Española estaban vestidos. El gusto por la ropa terminó por imponerse entre muchos, particularmente entre los caciques y sus capitanes.

Vestir a los indígenas se consideró parte del esfuerzo evangelizador, medio para apartarlos de una apariencia y un pensamiento salvaje, de actitudes pecaminosas (figura 1). El uso de ropa se convirtió en una marca de cristiandad entre indígenas y esclavos africanos. El rechazo a su empleo o la desnudez, servía para identificar a fugitivos y cimarrones, a gente fuera de la imagen requerida por el nuevo orden (Larrazabal 1975, p. 108 citado por Kulstad 2008, p. 267).

En los primeros contactos en Las Antillas y Yucatán, las prendas entregadas a los indígenas en intercambio o regalo incluyeron bonetes, jubones, sayos, zaragüelles y, sobre todo, camisas. El listado de la ropa entregada en Puerto Rico entre 1515 y 1517 al cacique de Caguas, sus capitanes, indios y naborias por su trabajo en la hacienda Real del Toa, incluye 115 camisas en distinto tipo de tejido, tres sayos, 38 caperuzas, dos bonetes, siete pares de enaguas, 46 paños de cabeza, 10 pares de alpargatas y 14 zaragüelles, además de un par de zapatos de cordobán, un cinto y algunos otros objetos (Tanodi, 1971).

Hay poca información sobre Cuba, aunque en 1524 -en su testamento- Diego Velázquez lega ropas a sus indios. Esta sería distribuida a través de los caciques para poder beneficiar a todos. Menciona
Figura 1. Dibujo del siglo XvI de mujer indígena del norte de Sudamérica. Tomado de The Pierpont Morgan Library (1996)

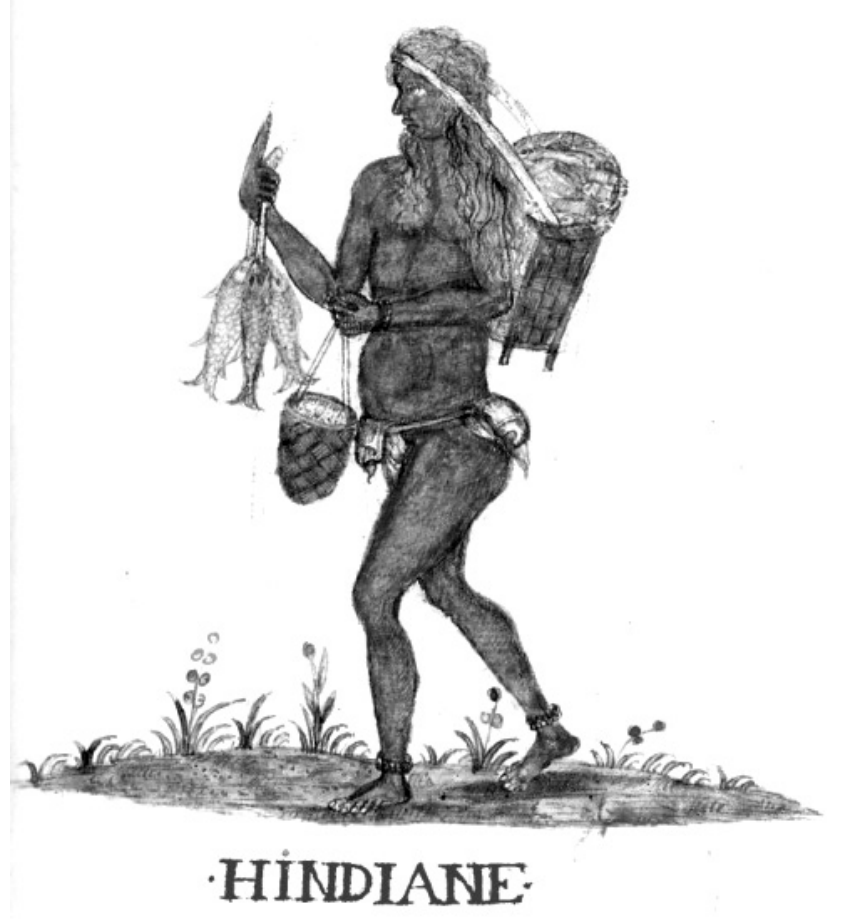

el vestuario como algo entregado de manera usual a los indios y especifica el nombre de ciertos indios y naborias favorecidos; indica dar sayos, zaragüelles, camisas y zapatos a los hombres, y camisas, enaguas, servillas y paños a las mujeres. La entrega por Gonzalo de Guzmán a sus indios de Bocas de Baní, de 50 camisas de presilla, confirma la tendencia vista en La Española y Puerto Rico, en torno al carácter básico de la camisa en el ajuar establecido para el indio. Zaragüelles y enaguas, como prendas para las piernas, completaban la vestimenta para el caso de hombres y mujeres, respectivamente. Estas se repartían con menos frecuencia, quizás por su mayor resistencia y durabilidad. Se dan también sayos, pero en poca cantidad y por su carácter de vestido a usar sobre la camisa, tal vez solo a los caciques. La presilla y otros tejidos bastos son los usados para confeccionar estas prendas.

Quizás algunos tipos de camisa fueran largas y se usaron como pieza única. Entierros con camisas 
Figura 2. Tela de lino, asociada posiblemente a elementos de vestuario; $\mathbf{2} \mathrm{cm}$ de largo el fragmento mayor. Encontrada en un entierro indígena del siglo XVI. Sitio El Chorro de Maíta, Cuba. Foto del autor.

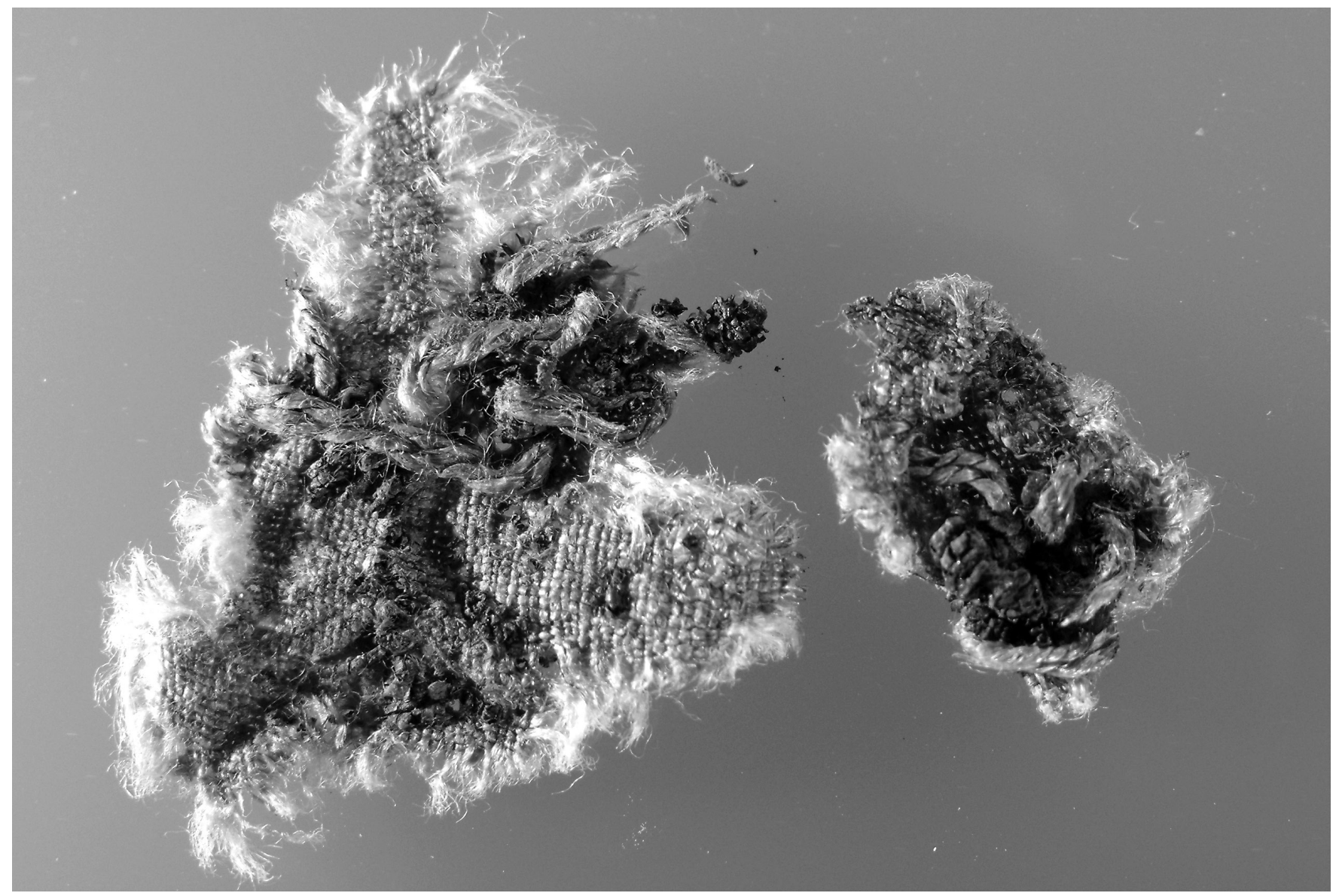

largas o con camisas, zaragüelles o enaguas, podrían generar los patrones tafonómicos de rellenamiento diferido identificados en las costillas, y en costillas y coxales de individuos indígenas en el cementerio del sitio arqueológico El Chorro de Maíta, en Cuba (figura 2) (Valcárcel Rojas, 2016).

En la Europa del siglo XVI sobre la camisa y las calzas se portaban varias prendas. Andar solo con camisa se consideraba estar desnudo y no era común ni decente, incluso entre pobres, aun cuando en el campo los labradores lo hicieran (Bernis, 1962). En el Caribe, muchas veces el clima obligó a los españoles a estar en camisa, pero no era apropiado y la tendencia era a vestir del mejor modo posible. El control de la ropa generó peleas entre los colonos y protegerlas del robo de piratas y corsarios fue toda una prioridad.

La posibilidad de la desnudez aproximaba a indígenas y europeos, y habría supuesto toda una crisis de civilidad y de cristiandad, de pérdida de control sobre los dominados. La desnudez fue excluida para ambos bandos. Náufragos y sobrevivientes de expediciones la mencionan tanto para ilustrar la desesperación real de los que vivieron estos hechos como el heroísmo que otros les atribuyen (Glantz, 2005). En cualquier caso aparece como algo antinatural, inherente a una situación límite que, a toda costa, se intenta evitar y es solo explicable en aquellos indios imposibles de civilizar y salvar. 
A los ojos europeos la desnudez de los hombres era signo de una naturaleza inferior $y$, la de la mujer indígena, evidencia de su disponibilidad para una sexualidad lujuriosa. La desnudez y su condición femenina las ponían fuera de cualquier consideración y respeto. Sobre ellas se volcó el lado reprimido de una moral hipócrita y de una falsa religiosidad, que las cosificó y vejó. Usar ropas no las puso a salvo de su destino de concubinas y sirvientas, de propiedades para el trabajo, los hijos y el sexo.

Vestir a los indios con las telas más pobres y las prendas mínimas, quedando desnudos según criterios occidentales, reproducía los códigos estamentales hispanos; colocaba al indígena en los estratos más bajos del ambiente colonial. Usar ropas implicaba además, la pérdida o fuerte atenuación de aquellos símbolos de identidad conectados con la desnudez, como el empleo de pinturas y ornamentos corporales. En 1566 el Concilio de Quito, en Ecuador, insistió en vestir a los indios y erradicar las pinturas corporales por su conexión con prácticas mágicas y heréticas (Patiño, 1990). El mal estaba en sus cuerpos desnudos o -quizás dirían los mismos indios-, vestidos de ancestros y mitos.

\section{El retorno masivo. Pacas en la Duarte}

La Duarte es una avenida que hoy sirve de eje $-y$ da nombre- a un peculiar universo comercial de la ciudad de Santo Domingo, esa que ayudaron a construir la gente de los caciques Yaguax y Caicedo. Las pacas de ropa de segunda mano son bultos pesados y coloridos, de cien libras de peso, que conviven con vendedores y dueños, amontonándose en almacenes o improvisados depósitos, tras haber cruzado algún mar u océano. De ellas brotan pantalones, vestidos, camisetas, blusas; se ordenan según su calidad en infinitas hileras o, principalmente, se amontonan para crear una peculiar orografía de pobreza. La paca es una especie de barco negrero donde la ropa es amontonada, comprimida, aplastada, para ser sacada y transportada más allá del mundo de sus usuarios originales. Se le ultraja física y espiritualmente. Es difícil pensar que alguna vez esa ropa relucía en una tienda, que muchas de esas ajadas prendas fueron miradas $\mathrm{y}$ tocadas por cientos de personas ponderadas, elogiadas; que alguien pudo ahorrar durante meses para hacerlas suyas o de otro, en un acto de lujuria consumista o bondad.

Las personas hunden sus manos en las montañas de vestuario de segunda mano, bucean tras la prenda adecuada, la elegida, la más barata, la que se ajusta a la ocasión o al bolsillo. Estratos de ropa se acumulan en los cajones para ser, finalmente, removidos cuando la posibilidad de vender se agota aunque muchas piezas deben permanecer allí, olvidadas, en un trasiego interminable de cosa desechada, de basura de primer mundo en un antro del tercero. ${ }^{2}$

Cuando visitas la Duarte, recuerdas los textos del arribo europeo en el siglo XV. La basura es cambiada por oro, se repite el acto comercial, ya sin exotismos y resignificaciones indígenas. Los viejos valores que ponderaban el brillo de la mayólica y las cuentas de vidrio, su origen lejano, su misterioso camino y a sus poderosos proveedores, mientras subestimaban el afeminamiento, la debilidad y cotidianeidad del oro, fueron sustituidos. Hace mucho se instauró un gusto homogeneizado, dictado por los conquistadores de siempre y por las necesidades de gente más pobre que sus ignorados ancestros de entonces, aun cuando transiten vestidos.

Acá hay una vieja camisa Perry Ellis, una sudadera Adidas, un pantalón Old Navy. En la capa formada por la ropa depositada la pasada semana abundan las de tallas XL, algo pasadas de moda, dejadas por jubilados que descansan muchos miles de kilómetros

\footnotetext{
2. Un cuidadoso análisis del comercio de ropa de segunda mano, su amplitud global y sus múltiples significados e implicaciones se puede encontrar en los trabajos de Andrew Brooks (2015), Hansen (2004a, 2004b) y Norris (2010), entre otros autores.
} 
Figura 3. Venta de ropas de segunda mano en la avenida Duarte, Santo Domingo, República Dominicana. Foto del autor.

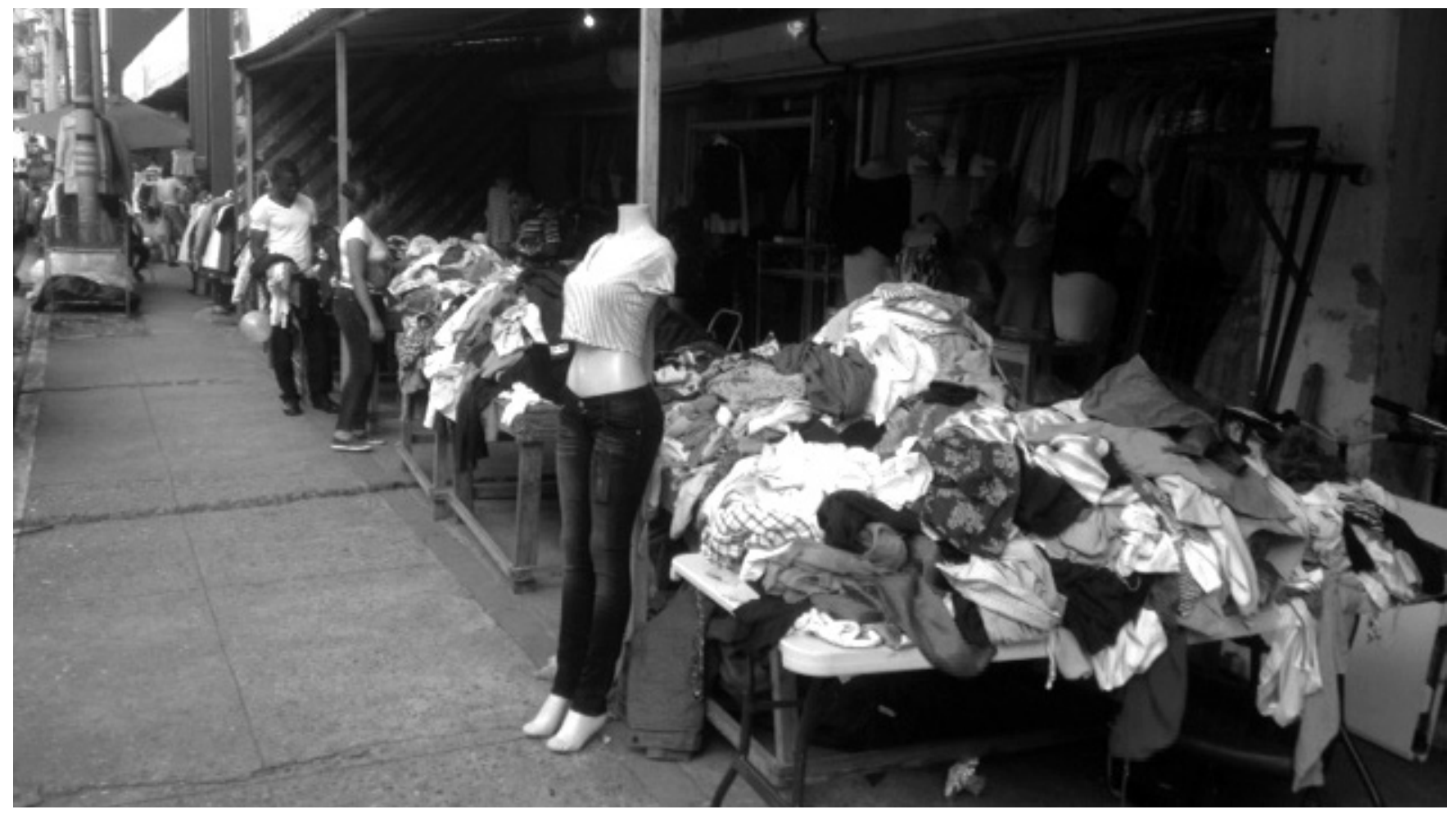

al norte. Las camisetas GAP de hace cinco años se conservan bien, fueron compradas por docena cuando alguien quiso cambiar su ropero, quizás feliz porque la economía de su país ya tenía mejor tono. En una esquina hay un feliz bolsón de trajes de baño, prácticamente nuevos, porque ese año el viaje a las playas de Portugal o a las Bahamas se canceló. La camiseta con la máscara de Spider-Man fue comprada en la Comic-Con del 2014, en San Diego. Es una talla $S$ y está muy gastada; era de un hombre delgado o de un muchacho; tal vez la usaron con intensidad y cariño, fue la Woody del armario, o sufrió los muchos cambios de estante en sucesivas tiendas de ropa de paca. Está muriendo ahora en una caja de cartón donde venden cosas a diez pesos, sucias, ajadas, miradas de refilón por la anciana cansada, de bolsa semivacía (figura 3).

Cavando en las ropas hay compradores de muchos tipos, mayormente gente de a pie, paleando sus necesidades, pero no todos son pobres de solemnidad.
Algunos van tras la ropa que, en tiendas regulares, cuesta diez o quince veces más; la moda y la estética internacional o la quimérica búsqueda de la diferencia en un mundo global nos alcanza. Ya no somos culpables de no tener ropa típica y resolvemos pareciéndonos a alguien, imaginándonos, flotando en la ficción que logramos pagar. La ropa es inocente y también quienes la necesitan; otra cosa es la vanidad y peor, el lucro.

$\mathrm{Al}$ igual que las cerámicas indígenas en las entrañas de los campos caribeños, las ropas gritan historias, pero no hay tiempo para escuchar ni oídos atentos. Llega otra paca y las prendas de cien pesos serán vendidas para que las comercien en otro lugar o viajarán al cajón de las de cincuenta pesos en una triste caída con final en los basureros de las afueras, consumidas como combustible en alguna industria, o quién sabe cómo. Los objetos se hacen cada día más anónimos, más usables, más fáciles de abandonar. Pasaron las épocas donde los pobres no 
podían tener ropas de ricos y aparentar un estatus inalcanzable, por su sangre, raza, color, nacimiento. Ahora, ocasionalmente y con un poco de suerte, el vendedor de plátanos puede aspirar a llevar una camisa dejada por el dueño de la tienda de autos, sin embargo, no engañará a nadie ni tendrá nunca ni una tuerca de su Porsche.

Hay otros símbolos y la masificación de la producción, su transnacionalización, ha abaratado mucho la indumentaria. La gente de Bangladesh fabrica mares de ropa para marcas de moda, mientras les pagan salarios absurdos y mueren en fábricas abarrotadas. Los pobres de allá cosen lo que los pobres o menos pobres de aquí, usarán cuando los ricos o menos ricos de dondequiera, decidan pasar a otro color, otra textura, un nuevo guiño de Zara, o una feliz ocurrencia de Calvin Klein. Se democratiza la estética en un desfase de ilusiones y en una carrera de olvidos que al final la pobreza rentabiliza porque la gente necesita vestirse, y los que tienen no dejan de crear formas para ganar más dinero. Vender la basura a los del sur o a los pobres del norte, es una de ellas. Se debe dejar espacio para lo verde, la vida salvaje y las mascotas, aunque la inmundicia finalmente termine subiendo porque el planeta, $y$ perdonen la machacona repetición, es uno solo.

Hay mucha ropa donada, movida al comercio, o de ventas inicialmente hechas para conseguir fondos humanitarios. De cualquier modo, la marea de lo usado avanza, unificando nuestra materialidad $y$, en alguna medida nuestra apariencia, en un proceso donde el simbolismo material del poder busca nuevos posicionamientos. En la loma de vestidos se encuentran las maquiladoras de Latinoamérica y Asia; hay sangre de niño de la India y los recuerdos de un muchacho de Dublín. Está la buena fe de una anciana de Florida y se coló una playera de aquel actor junto al pantalón que un haitiano agradecido $-y$ casi indiferente a la solidaridad internacional- vendió en su negocio de la frontera con República Dominicana.
Los textiles cosidos, presillados, remachados, se trafican en pacas que son abiertas en muchos lugares del Caribe y el mundo. Encarnan -como nunca antes- nuestras interconexiones, universos y destinos, diciéndonos que somos uno solo $\mathrm{y}$, a la vez, múltiples y diversos. Las ropas nuevas y viejas, desechadas, movidas en las pacas, rompen y crean diferencias, pero no son usadas por sus primeros compradores, aunque puede que -casi harapos- vuelvan a la muchacha latina que las cosió. Seguimos teniendo dos bandos en eso de producir, poseer y consumir.

La Duarte es una avenida oscura, bordeada de basura y frágiles puestos de venta, surcada por un río interminable de automóviles, motos, carros abollados y gente. Una más en el dominante lado pobre del planeta. Ese orina a la vista de todos, unos comen y otros anuncian sus productos; aquellos piden limosnas, el "pelo malo" se enmascara tras mechones de lugares inimaginables, se negocia o sugiere algún servicio sexual, la mayoría intenta vender o comprar al mejor precio, se trabaja hasta el cansancio, se vislumbra una pistola, la lluvia llega de repente, magnífica y tropical, y deja una línea de costra negra en las aceras, un mar de bolsas plásticas. Todos saben que las pacas vienen de Estados Unidos, Europa, Corea del Sur. Es un negocio de cientos de miles de toneladas métricas y millones de dólares, preocupante para productores, exportadores y vendedores de ropa, que da trabajo a muchas personas. Del ambiente deprimente y caótico de la Duarte brota dinero; de seguro, muy poco permanece allí.

Los chinos vigilan desde cámaras de video y altos mostradores a los muchos empleados mal pagados en sus tiendas abarrotadas, rodeados por mercancía barata y de corta vida. Los venezolanos venden arepas, los cubanos compran en una batalla perdida contra el monopolio comercial de su gobierno, los haitianos están dondequiera y hacen cualquier cosa... los emigrantes nadan en los entornos de la marginalidad, en un flujo bullicioso, buscando esa 
soñada mejor vida que la República Dominicana está ofreciendo. Ni las manufacturas chinas, omnipresentes en la Duarte, detienen a las pacas.

El mercado de Santo Domingo vive un segundo tiempo de multiculturalidad, protagonismo americano y viajes transoceánicos, solo que la carga no está en la ciudad vieja ni es oro o perlas en ruta a Europa, como en el siglo XVI. Son ropas que van quedando en el camino, tras el ejercicio de poder del capital global, lo adictivo de las modas y la desesperanza de ocultar el cuerpo, pese a ser -el nuestro- el mejor de los climas.

\section{Al final, las ropas}

Las ropas para indios, producidas en Europa, entraron al mismo circuito comercial que movía hacia el Viejo Mundo el oro de ríos y minas antillanas. El entorno global iniciado en aquellos ańos halló en las necesidades del asentamiento colonial, una motivación para impulsar las manufacturas; alimentó el consumo de pueblos donde se querían replicar las ciudades de sus fundadores y el fausto de lejanas cortes, y reafirmar viejas y nuevas diferencias. La ropa dio a los indios la imagen y condición requerida en el plan de dominación, ayudó a aplastar su identidad originaria y a esconder su memoria en el universo de pobreza que construían los nuevos tiempos de centralidad occidental. Los incorporó al viejo ciclo europeo de cuerpos vestidos y almas desnudas.

Ese ciclo perdura y la contracción del tiempo y espacio que produjo la integración de América al mundo conocido, desatando la globalización, se reafirma usando los símbolos de un consumo que fue desarrollándose desde el "Descubrimiento". Las ropas vuelven de lugares lejanos para construir al Otro, para cubrirlo y encubrirlo, en los complejos bordes de una realidad que no podemos dejar de mirar.
Si la categoría de indio suplantó y homogeneizó los viejos etnónimos, suprimiendo términos como ciguayo, macorix, lucayo, haciendo del vestuario un recurso de enmascaramiento y borrador de identidades y culturas, el mundo de la ropa de segunda mano, de las pacas, hace más cercanos a dominicanos, haitianos, cubanos y venezolanos. Los coloca de un mismo lado en la demarcación de la riqueza, contribuye a conectarlos y a romper sus diferencias nacionales, sus estatus migratorios, sus perfiles étnicos, mientras interactúan en la Duarte, en un mismo río de pobreza.

\section{Agradecimientos}

Los resultados que se presentan fueron obtenidos como parte de las investigaciones desarrolladas por el autor en el proyecto ERC-Synergy NEXUS 1492, sostenido por European Research Council / ERC grant agreement $\mathrm{n}^{\circ} 3192099$. El texto fue preparado durante la estancia de investigación posdoctoral que, en el contexto del proyecto NEXUS, desarrolla el autor en el Instituto Tecnológico de Santo Domingo, República Dominicana, y se inserta en el trabajo del Grupo de Investigación "Arqueología y culturas del Caribe", adscrito a esta Institución.

\section{Referencias}

Bernis, C. (1962). Indumentaria Española en tiempos de Carlos V. Madrid: Instituto Diego Velázquez, del Consejo Superior de Investigaciones Científicas.

Brooks, A. (2015). Clothing Poverty: The Hidden World of Fast Fashion and Second-Hand Clothes. London: Zed Books.

Las Casas, Bartolomé de (1875). Historia de Las Indias (Vol. t.1). Madrid: Imprenta de Miguel Ginesta. 
Dussel, E. (1994). 1492. El Encubrimiento del otro. Hacia el origen del "mito de la modernidad". La Paz: Plural editores.

Glantz, M. (2005). La desnudez como naufragio. Madrid: Iberoamericana.

Hansen, K. T. (2004a). Helping or hindering? Controversies around the international second-hand clothing trade. Anthropology Today 20(4), 3-9.

Hansen, K. T. (2004b). The World in Dress: Anthropological Perspectives on Clothing, Fashion, and Culture. Annual Review of Anthropology, 33, 369-392.

Kulstad, P. M. (2008). Concepcion de La Vega 1495-1564: A preliminary look at lifeways in the americas' first boom town. Unpublished Master tesis, University of Florida, Gainesville.

Larrazábal, C. (1975). Los negros y la esclavitud en Santo Domingo. Santo Domingo: J.D. Postigo.

Mira Caballos, E. (1997). El Indio Antillano: repartimiento, encomienda y esclavitud (1492-1542). Sevilla: Muñoz Moya Editor.

Mira Caballos, E. (2000a). Las Antillas Mayores 1492-1550. Ensayos y documentos. Madrid: Iberoamericana.

Muro Orejón, A. (1956). Ordenanzas reales sobre los indios. (Las Leyes de 1512-13), transcripción, estudio y notas. Anuario de estudios americanos (13), 417- 471.

Norris, L. (2010). Recycling Indian Clothing: Global Contexts of Reuse and Value. Bloomington: Indiana University Press.

Patiño, V. M. (1990). Historia de la Cultura Material en la América Equinoccial. Vestidos, adornos y vida social (Tomo 4). Bogotá: Instituto Caro y Cuervo.

Tanodi, A. (1971). Documentos de la Real Hacienda de Puerto Rico (Vol. I (1510-1519). Río Piedras: Centro de Investigaciones Históricas. Universidad de Puerto Rico.

The Pierpont Morgan Library (1996). Histoire Naturelle des Indes: The Drake Manuscript in The Pierpont Morgan Library. London.

Valcárcel Rojas, R. (2016). Archaeology of Early Colonial Interaction at El Chorro de Maita, Cuba. Gainesville: University Press of Florida.

\section{Datos de filiación}

Roberto Valcárcel Rojas. Doctor en Arqueología por la Universidad de Leiden donde actualmente se desempeña como investigador posdoctoral adscrito al proyecto ERC Sinergy-NEXUS 1492. Profesor invitado del Instituto Tecnológico de Santo Domingo. Sus intereses de investigación incluyen el estudio de las sociedades indígenas del Caribe y los procesos de interacción cultural en la América colonial, combinando datos arqueológicos e históricos. Es autor del libro Archaeology of Early Colonial Interaction at El Chorro de Maita, Cuba (2016), editor principal de Un rostro local para la arqueología cubana (2016), junto a José Abreu Cardet, y coeditor, junto a Jorge Ulloa Hung, de Indigenas e Indios en el Caribe. Presencia, Legado y Estudio (2016). Ha publicado artículos, como autor principal o coautor, en International Journal of Historical Archaeology, Journal of Anthropological Archaeology, Journal of Archaeological Science, y Latin American Antiquity, entre otros.

E-mail: rv.rojas68@yahoo.es 